\title{
Regularized singular boundary method for groundwater flow in a cofferdam
}

\author{
Juraj Mužík ${ }^{1, *}$, and Roman Bulko ${ }^{1}$ \\ ${ }^{1}$ University of Žilina, Department of geotechnics, Univerzitná 8215/1, 01026 Žilina, Slovakia
}

\begin{abstract}
The boundary integral methods form one group of methods for solving differential equations. The boundary element method (BEM) is the basic method of this group. However, it requires the boundary mesh of elements and the evaluation of improper singular integrals, that arise due to fundamental solution singularity. Therefore, boundary meshless methods have recently have come into focus to remove these shortcomings. One of the most promising boundary collocation numerical schemes is the singular boundary method (SBM). To tackle the singularity of the fundamental solution, this method adopts the concept of original intensity factors (OIFs). The application of the proposed SBM scheme to groundwater flow in the cofferdam structure is presented and compared to the finite element method (FEM) solution.
\end{abstract}

\section{Introduction}

The boundary element method (BEM) is one of those modern numerical methods whose applications can be found in all the technical disciplines as well as in the natural sciences. One of the most effective applications of the BEM is its use for the solution of groundwater flow problems encountered in the design and maintenance of hydraulic structures and tailing dams, in soil mechanics, hydrology, hydrogeology and engineering geology

BEM represents the family of the methods based on the boundary integral theory [1]. However, the BEM is an imposing method from the mathematical point of view and also time-consuming for numerical integration. Despite its popularity, there are many problems concerning its efficient implementation, especially the difficulty in meshing surfaces and the need to compute complicated singular integrals.

Therefore some new boundary meshless methods are developed which would alleviate these problems. The method of fundamental solutions (MFS) [2-4] overcomes singularities of BEM using a fictitious boundary but the optimum location of this boundary remains the open problem especially for the complex-shaped domains. To avoid the fictitious boundary of MFS, Chen $[5,6]$ proposed a new numerical method called a singular boundary method (SBM). In the SBM the main diagonal members of the characteristic matrix are evaluated using the concept of origin intensity factors (OIF) [5]. The OIF for the Neumann boundary condition is usually solved using the subtracting and adding-back regularization [7] but the OIF for Dirichlet boundary conditions is the weak point of the SBM. Several techniques

\footnotetext{
* Corresponding author: muzik@fstav.uniza.sk
} 
have been developed to determine these OIFs, namely, inverse interpolation technique (IIT), empirical approaches [7] based mainly on the fundamental solution integrals mean value [4]. In order to resolve the Dirichlet boundary condition OIFs in a rigorous way, we applied the regularized boundary integral formulation described in [8]. The accuracy and efficiency of this method are verified in the numerical experiment of steady-state groundwater flow in cofferdam.

\section{Governing equations}

The 2D groundwater steady-state flow can be described by Laplace equation

$$
\Delta h(\mathbf{s})=0 \quad \mathbf{s} \in \Omega
$$

with boundary conditions

$$
\begin{aligned}
& h(\mathbf{s})=g(\mathbf{s}) \quad \mathbf{s} \in \Gamma_{D} \\
& \frac{\partial h(\mathbf{s})}{\partial n(\mathbf{s})}=f(\mathbf{s}) \quad \mathbf{s} \in \Gamma_{N}
\end{aligned}
$$

where $h$ is the groundwater head. The methods based on the boundary integral theory represent $h$ in the term of the single layer potential as

$$
h(\mathbf{s})=\int[G(\mathbf{x}, \mathbf{s}) \sigma(\mathbf{x}) d \Gamma
$$

which gives the equations [7]

$$
\begin{aligned}
& \int_{\Gamma_{D}} G(\mathbf{x}, \mathbf{s}) \sigma(\mathbf{x}) d \Gamma=g(\mathbf{s}) \quad \mathbf{s} \in \Gamma_{D} \\
& \int_{\Gamma_{N}} \frac{\partial G(\mathbf{x}, \mathbf{s})}{\partial n(\mathbf{s})} \sigma(\mathbf{x}) d \Gamma=f(\mathbf{s}) \quad \mathbf{s} \in \Gamma_{N}
\end{aligned}
$$

where $G(\boldsymbol{x}, \boldsymbol{s})$ is the fundamental solution of the 2D Laplace equation and $\sigma(\boldsymbol{x})$ is the unknown density in the point $\mathbf{x}$. The fundamental solution of the two-dimensional Laplace equation is

$$
G(\mathbf{x}, \mathbf{s})=-\frac{1}{2 \pi} \ln (\|\mathbf{s}-\mathbf{x}\|)
$$

Equations (3) and (4) are the basis of all boundary oriented methods. BEM applies discretization of the boundary $\Gamma$ to particular elements; MFS and SBM use the numerical approximation of the integrals (details see e. g. in [4]). Then (3) is changed to

$$
\begin{aligned}
& h\left(\mathbf{s}_{i}\right)=\sum_{j=1}^{n} G\left(\mathbf{x}_{j}, \mathbf{s}_{i}\right) \alpha_{i j} \quad \mathbf{x}, \mathbf{s} \in \Gamma \\
& \frac{\partial h\left(\mathbf{s}_{i}\right)}{\partial n\left(\mathbf{s}_{i}\right)}=\sum_{j=1}^{n} \frac{\partial G\left(\mathbf{x}_{j}, \mathbf{s}_{i}\right)}{\partial n\left(\mathbf{s}_{i}\right)} \alpha_{i j}
\end{aligned}
$$


When $\mathbf{s} \equiv \mathbf{x}$ the fundamental solution $G(x, s)$ tends to infinity and therefore the SBM introduces the concept of origin intensity factors. The equations (6) can now be written as

$$
\begin{aligned}
& h\left(\mathbf{s}_{i}\right)=\sum_{j=1, j \neq i}^{n} G\left(\mathbf{x}_{j}, \mathbf{s}_{i}\right) \alpha_{i j}+U_{i i} \alpha_{i i} \\
& \frac{\partial h\left(\mathbf{s}_{i}\right)}{\partial n\left(\mathbf{s}_{i}\right)}=\sum_{j=1, j \neq i}^{n} \frac{G\left(\mathbf{x}_{j}, \mathbf{s}_{i}\right)}{\partial n\left(\mathbf{s}_{i}\right)} \alpha_{i j}+Q_{i i} \alpha_{i i}
\end{aligned}
$$

where $U_{i i}, Q_{i i}$ is the Dirichlet and Neumann origin intensity factors, respectively.

\section{Singularity treatments - origin intensity factors}

Origin intensity factors (OIFs) are a key problem for the SBM. While the OIFs for Neumann's boundary conditions have long been satisfactorily derived using a subtracting and adding-back technique [7], factors for the Dirichlet condition are still under development. Various variants of inverse interpolation techniques (IIT) [7], empirical formulas [10], and formulas using surface integrals of the fundamental solution [4] are used for their determination. All of these variants increase the complexity of this method. In order to circumvent the singularity of the Dirichlet OIF we used the following integral identity

$$
\int_{\Gamma} n(\mathbf{s}) G(\mathbf{x}, \mathbf{s}) d \Gamma=\int_{\Gamma}(\mathbf{s}-\mathbf{x}) \frac{\partial G(\mathbf{x}, \mathbf{s})}{\partial n(\mathbf{x})} d \Gamma
$$

to get regularized boundary integral equation instead of (3) (see [8])

$$
\begin{aligned}
& h(\mathbf{s})=\int_{\Gamma}[\sigma(\mathbf{x})-\sigma(\mathbf{s}) \mathbf{n}(\mathbf{s}) \cdot \mathbf{n}(\mathbf{x})] G(\mathbf{x}, \mathbf{s}) d \Gamma \\
& +\sigma(\mathbf{s}) \int_{\Gamma} \mathbf{n}(\mathbf{s}) \cdot(\mathbf{x}-\mathbf{s}) \frac{\partial G(\mathbf{x}, \mathbf{s})}{\partial \mathbf{n}(\mathbf{x})} d \Gamma
\end{aligned}
$$

This equation can be also written as

$$
h(\mathbf{s})=\int_{\Gamma} G(\mathbf{x}, \mathbf{s}) \sigma(\mathbf{x}) d \Gamma-\sigma(\mathbf{x})\left[\begin{array}{l}
\int_{\Gamma} \mathbf{n}(\mathbf{s}) \cdot \mathbf{n}(\mathbf{x}) G(\mathbf{x}, \mathbf{s}) d \Gamma \\
-\int_{\Gamma} \mathbf{n}(\mathbf{s}) \cdot(\mathbf{x}-\mathbf{s}) \frac{\partial G(\mathbf{x}, \mathbf{s})}{\partial \mathbf{n}(\mathbf{x})} d \Gamma
\end{array}\right]
$$

Starting from (7) and using the weighted diagonal elements the numerical approximation of integrals in (10) brings on the following expression

$$
h\left(\mathbf{s}_{i}\right)=\sum_{j=1, j \neq i}^{n} G\left(\mathbf{x}_{j}, \mathbf{s}_{i}\right) \alpha_{i j}-\frac{\alpha_{i i}}{L_{i}}\left[\begin{array}{c}
\sum_{k=1, k \neq i}^{n} L_{k} \mathbf{n}\left(\mathbf{s}_{i}\right) \cdot \mathbf{n}\left(\mathbf{s}_{k}\right) G\left(\mathbf{x}_{k}, \mathbf{s}_{i}\right)- \\
\sum_{k=1, k \neq i}^{n} L_{k} \mathbf{n}\left(\mathbf{s}_{i}\right) \cdot\left(\mathbf{x}_{k}-\mathbf{s}_{i}\right) \frac{G\left(\mathbf{x}_{k}, \mathbf{s}_{i}\right)}{\partial n\left(\mathbf{x}_{k}\right)}
\end{array}\right]
$$


where $L_{i}$ is the length of boundary section in the vicinity of the point $i$ on the boundary $\Gamma$ (see Figure 1).

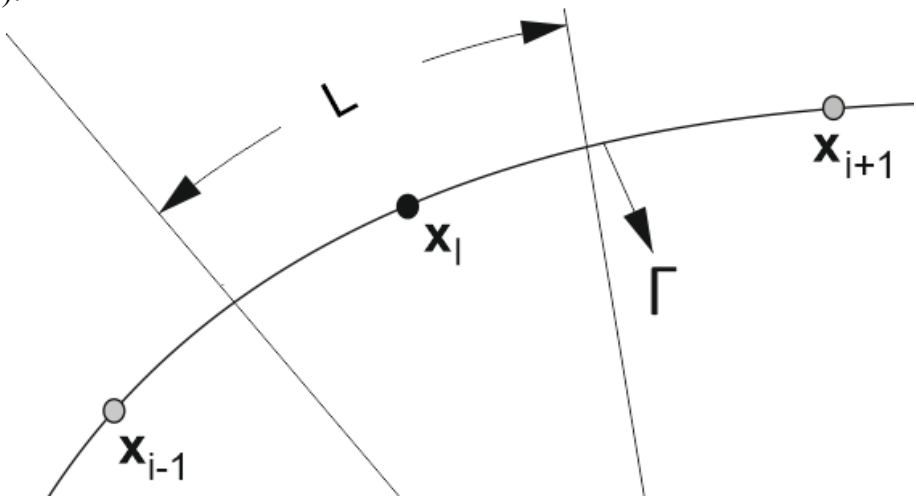

Fig. 1. Description of boundary part L around collocation point $x_{i}$.

The Dirichlet OIF can now be written as

$$
U_{i i}=-\frac{1}{L_{i}} \sum_{k=1, k \neq i}^{n} L_{k}\left[\mathbf{n}\left(\mathbf{s}_{i}\right) \cdot \mathbf{n}\left(\mathbf{x}_{k}\right) G\left(\mathbf{x}_{i}, \mathbf{s}_{k}\right)-\mathbf{n}\left(\mathbf{s}_{i}\right) \cdot\left(\mathbf{x}_{k}-\mathbf{s}_{j}\right) \frac{\partial G\left(\mathbf{x}_{k}, \mathbf{s}_{i}\right)}{\partial n\left(\mathbf{x}_{k}\right)}\right]
$$

It is worth noting that the described procedure is valid not only for two dimensional but also for three-dimensional potential problems and it could effectively solve the problem with the Dirichlet OIF.

\section{Numerical example - Coffer Dam flow}

In this section, a numerical example of groundwater flow in a cofferdam is presented to illustrate the accuracy and effectivity of the code based on the proposed regularized SBM scheme. Results are compared with the solution obtained by finite element method and root-mean-square errors (RMSE) has been computed as

$$
\text { RMSerror }=\sqrt{\frac{\sum_{i}\left(f_{i}-a_{i}\right)^{2}}{\sum_{i} a_{i}^{2}}}
$$

where $a_{i}$ are the exact values and $f_{i}$ are those computed using the proposed numerical scheme. The implementation uses a Visual $\mathrm{C}++$ programming language with multithreaded OpenBLAS 0.2 .20 on an Intel Core(TM) i7-6500U processor running at $2.5 \mathrm{GHz}$ workstation having a total of $16 \mathrm{~GB}$ memory.

A numerical example is based on problem 2.3 from []. A riverbed consists of a layer of sand $10 \mathrm{~m}$ thick overlying impermeable rock: the depth of water is $3 \mathrm{~m}$. A long cofferdam 5 $\mathrm{m}$ wide is formed by driving two lines of sheet piling to a depth of $5 \mathrm{~m}$ below the level of the river bed and excavation to a depth of $2.50 \mathrm{~m}$ below bed level is carried out within the cofferdam. The water level within the cofferdam is kept at excavation level by pumping. The coefficient of permeability of the sand subsurface is $K_{x}=K_{y}=2.6 \times 10^{-5} \mathrm{~ms}^{-1}$. The problem was solved by FEM (Seep/W) (see. Figure 2 and Figure 3) and using SBM code (see Figure 4, Figure 5, and Figure 6). The solution obtained by SBM showed a superiority over the one of FEM, the results showed higher smoothness of SBM solution, and overall 
difference between computed groundwater head surfaces by means of (13) RMSerror $=$ $2.698 \times 10^{-3}$.

The computational effort for the FEM (in the configuration described by Figure 2) was 312 equations (sparse matrix) compared to 122 equations of SBM formulation. The characteristic matrix for SBM was created for each one of the 3 rectangular domains (see Figure 4) and at the connection points, compatibility and continuity conditions apply.

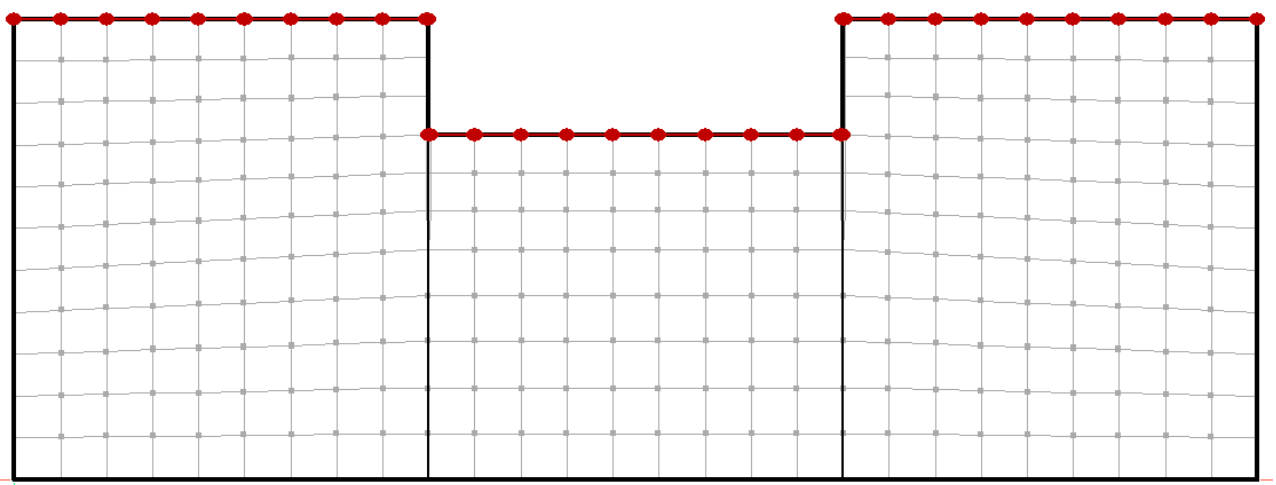

Fig. 2. FEM mesh (4-node quadrilateral elements) used for calculation.

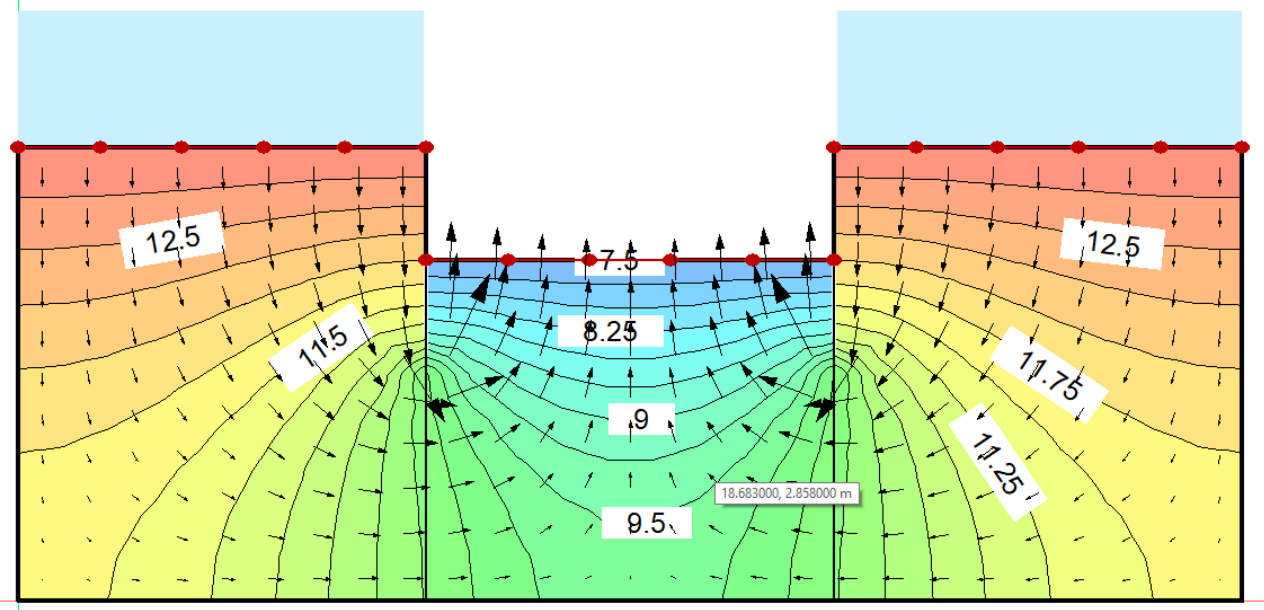

Fig. 3. FEM calculation results - the velocity vectors and groundwater head contours. 


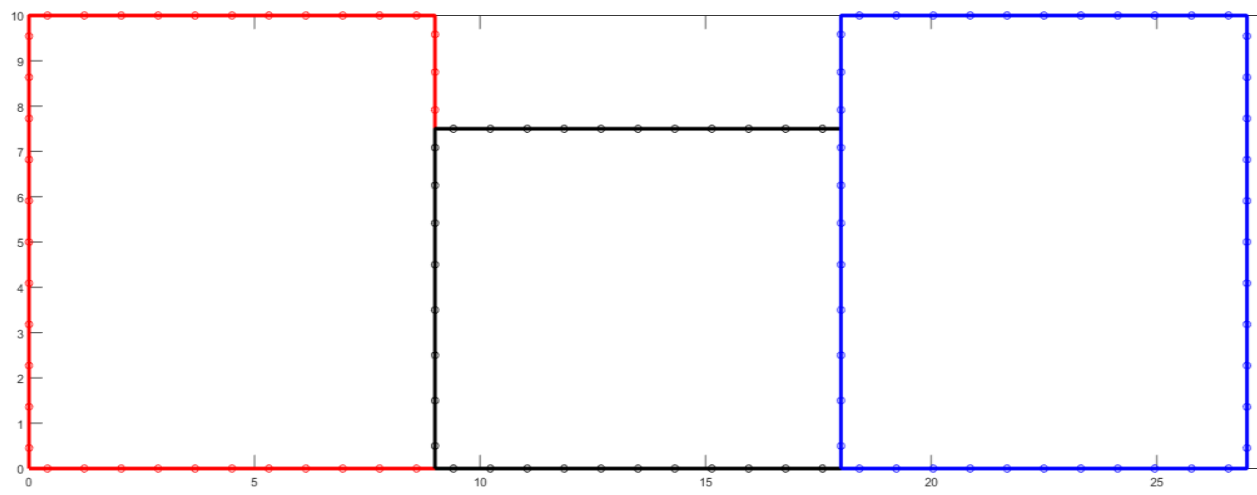

Fig. 4. SBM nodal configuration of the model.

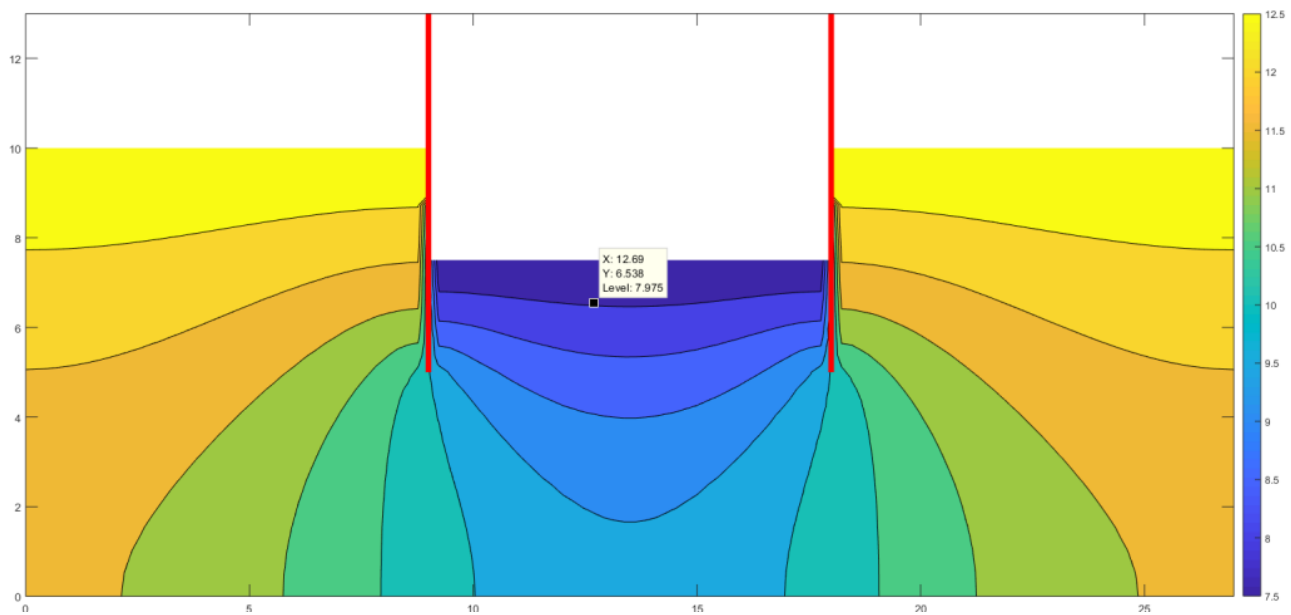

Fig. 5. SBM - groundwater head contours.

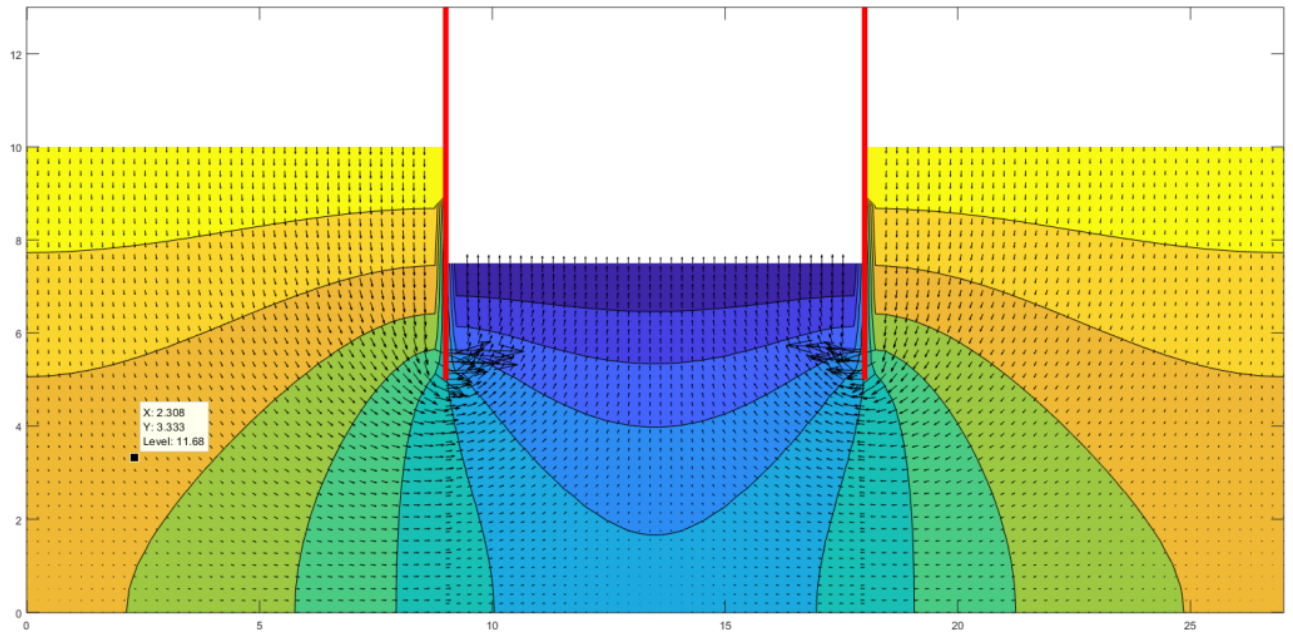

Fig. 6. SBM - groundwater head contours and velocity vectors calculated using numerical gradient. 


\section{Conclusions}

This article is focused on the groundwater flow problem in cofferdam performed using new regularized SBM numerical scheme. Using regularization, we derived a relationship for Dirichlet origin intensity factors, and we tested this SBM scheme on a ground engineering boundary value problem that posses Dirichlet and homogeneous Neumann conditions. We compared the results with the FEM solution as well as the comparison of the computational cost of both methods. The comparison showed that the presented solution shows low errors and is cost-effective. In the near future, it will be necessary to verify the possibilities of this solution for solving anisotropic problems and also time-dependent problems.

This contribution is the result of the project funded by the Scientific Grant Agency of Slovak Republic (VEGA) No. 1-0716-17

\section{References}

1. C. Brebbia, J. Telles, L. Wrobel, Boundary Element Techniques, Gao li, Taipei, 1985

2. M. Golberg, Engineering Analysis With Boundary Elements, 16 (1995)

3. F C. Chen, A. Karageorghis, Y. Li, Numerical Algorithms, 72 (2015)

4. B. Šarler, Engineering Analysis With Boundary Elements, 33 (2009)

5. X. Wei, W. Chen, L. Sun, B. Chen, Engineering Analysis With Boundary Elements, 58 (2015)

6. Y. Gu, W. Chen, C. Zhang, International Journal Of Solids And Structures, 48 (2011)

7. K. Kováŕík, J. Mužík, R. Bulko, D. Sitányiová, Engineering Analysis With Boundary Elements, 83 (2017)

8. W. Qu, Y. Zhang, C. Liu, Advances In Engineering Software, 96 (2016) 